\title{
Exercise-Induced Growth in Golden Hamsters: Effects of Age, Weight, and Activity Level'
}

\author{
KATARINA TOMLJENOVIĆ BORER AND LINDA R. KAPLAN \\ Neuroscience Laboratory and Department of Psychology \\ University of Michigan, Ann Arbor, MI 48109
}

(Received 5 March 1976)

\begin{abstract}
BORER, K. T. AND L. R. KAPLAN. Exercise-induced growth in golden hamsters: effects of age, weight, and activity level. PHYSIOL. BEHAV. 18(1) 29-34, 1977. - The relationship between the exercise-induced growth and the endogenous controls of growth was examined in 356 golden hamsters of which equal numbers were given access to horizontal disc exercisers and maintained sedentary at different ages (17 and 42 days) and body weights $(25-180 \mathrm{~g})$. Hamsters started exercising around Day 35, increased their activity levels to 30,000 revolutions per day (RPD) by Day 35 , and ran progressively less as their weight increased above $65 \mathrm{~g}$. Exercise accelerated growth only in hamsters which have entered the slow asymptotic phase of growth, which weighed between 60-180 g and generated over 15,000 RPD: Disc exercise appears to reinstate higher rates of ponderal and linear growth after hamsters have entered the slow asymptotic phase of growth. Sustained inhibition of exponential growth may participate in the long-term regulation of body size in adult rodents.
\end{abstract}

Voluntary exercise Exponential growth Asymptotic growth Weight regulation Ontogeny

IN rodents and some other animals, linear and ponderal growth progresses through an early exponential phase followed by a slow asymptotic phase. The onset of asymptotic growth appears to be triggered by some influence of increasing body size. The inflexion from exponential to asymptotic growth takes place when mice [15] and rats $[8,9]$ attain a critical body mass. Thus young rodents whose early growth is exceptionally rapid enter the asymptotic phase of growth earlier than those rats $[8,19]$ and mice [15] whose early growth was retarded. Kennedy proposed that a change in metabolism or heat-transfer properties of the body at some critical body size may trigger changes in growth rate [8] and initiate some adult homeostatic functions such as puberty $[10,19]$ and weight regulation $[8,9,20]$.

Throughout the asymptotic phase of growth the rates of ponderal and linear growth appear to be inversely related to body size. This progressive decline in rodent growth rates as a function of increasing body size is not entirely due to a loss of tissue capacity for rapid ponderal and linear growth. The epiphyseal growth centers remain open in sexually mature rats [4], and the growth of rat skeleton proceeds throughout the second year of life [5]. Latent capacity for rapid skeletal and ponderal growth can be demonstrated in adult female rats by producing a partial deafferentation of the medial basal hypothalamus. When some of the connections of the medial basal hypothalamus of the female rat are transected by placing semicircular cuts anterior to this area $[12,13,17]$ or parasagittal cuts lateral to this area
[6], there is a reinstatement of rapid linear and ponderal growth, an excessive accumulation of body fat $[6,13,17]$. and increase in the levels of circulating growth hormons [12]. Growth-inducing knife cuts are not effective in young rats which are still growing at exponential rates $[5 \mid$. The asymptotic growth rate of adult rodents would, therefore. appear to result, in part, from some sustained inhibitory influence over the mechanisms of growth acting on the medial basal hypothalamic area from the anterolateral direction.

A recent discovery of a simple noninvasive technique for reverting the asymptotic growth rates in adult rodents 10 exponential ones has provided the opportunity for further study of the nature of the restraints exerted over linear and ponderal growth. If adult, slowly-growing hamsters are given the opportunity to exercise on horizontal activity dises they engage in high levels of voluntary activity and reenter the exponential phase of linear and ponderal growth throughout their exposure to such exercise [2]. Seviral days after the cessation of exercise the retired hamsters resume their slow asymptotic growth and engage in the normal defense of their increased body size [3]

In the present study we have determined the ages and body sizes at which hamsters first start exercising on discs and at which disc activity first accelerates growth. We have found an inverse relationship between the levels of voluntary disc activity and body size and have defined a linite range of activity levels which are compatible with accelerition of linear and ponderal growth in hamsters.

'This work was supported by a grant from The Weight Watchers Foundation, Inc. to K. T. Borer. We thank Elliot S. Valenstein fur the encouragement and for the use of laboratory facilities. Robert P. Kelch and Otto Sellinger made helpful suggestions for the improvemen of
the manuscript. 


\section{METHOD}

\section{Animals}

Golden Syrian hamsters (Mesocricetus auratus Waterhouse) were obtained from commercial breeders (Con Olsen, Madison WI and Engle Laboratory Animals, Farmersburg, IN). Three hundred fifty-six hamsters were studied. Of those, 86 were born in the laboratory and 270 were obtained at ages above 6 weeks. Hamsters older than 5 weeks were individually housed in standard suspended wire-bottom cages at all times except when assigned to exercise. During the exposure to disc exercise hamsters were individually housed in Plexiglas boxes containing a freely-turning horizontal Plexiglas disc, $23-25 \mathrm{~cm}$ in dia. [2]. Litters of hamsters were culled to 6 pups within the first week following birth and were housed in suspended cages twice the normal width with sawdust on solid floors. Purina laboratory chow and water were available at all times. Laboratory-reared and some of the adult hamsters also had unlimited supplies of sunflower seeds. Adult hamsters with access to sunflower seeds were used in studies, to be reported elsewhere, on the effects of exercise on dietary selection. The availability of sunflower seeds exerts no discernible effect over the exercise-induced growth [2]. Throughout the study animals were kept at $20-22^{\circ} \mathrm{C}$ and in $12 \mathrm{hr}$ of light beginning at $9 \mathrm{a} . \mathrm{m}$.

\section{Experimental Design and Measurements}

Hamsters were assigned to sedentary and exercising groups on the basis of equivalent body weights at the start of the experiment as well as by equivalence of birthweights in the case of laboratory-reared hamsters. Hamsters were weighed daily to determine the relative effects of exercise and sedentary existence on ponderal growth rates. The effects of exercise on linear growth was evaluated by taking the body length measurements of the laboratory-reared hamsters on the last day of the experiment. Length was measured between the tip of the nose and the tip of the tail in anesthetized hamsters which were extended with their ventral surface down over a $\mathrm{cm}$ scale. The percentage of body fat was estimated from the total body water content of hamsters which bears a constant relationship of $72.3 \%$ to the lean body mass in hamsters [11] and a variety of other animals [16]. It is assumed that this relationship does not change during and following exercise-induced weight displacements. Manipulations which affect body fat stores do no alter the water content of lean body mass in rats [1]. Body water determinations were made by drying the hamster carcasses to constant weight at temperatures between $30^{\circ}$ and $50^{\circ} \mathrm{C}$.

The onset of voluntary disc activity and changes in activity levels as a function of age were studied in two groups of laboratory-reared hamsters. Thirty-five hamsters were given access to discs at age 14-19 days for a 32-day period, while 30 sedentary animals served as controls. Twenty exercising hamsters and 19 sedentary hamsters were females, the remainder were males. Continuous access of young hamsters, but not of their lactating mothers, to disc exercisers were made possible through the design of communal boxes they were housed in. Communal boxes, $31 \mathrm{~cm}$ wide, $40 \mathrm{~cm}$ high, and $76 \mathrm{~cm}$ long were subdivided into a $23 \mathrm{~cm}$ long family compartment housing food, water, and lactating females and a $53 \mathrm{~cm}$ long playground area containing three stationary or freely-moving disc exercisers,
$15 \mathrm{~cm}$ in dia. A Plexiglas partition hearing three openimg it its base, $1.6 \mathrm{~cm}$ in dia. and $2 \mathrm{~cm} \mathrm{high,} \mathrm{allowed} \mathrm{a} \mathrm{litter} \mathrm{of} \mathrm{vi}$. young hamsters free access to both compartman! liu: prevented the lactating female fron reaching the diss. I: moveable dises were equipped with microswitches for ihs electric counting of disc revolutions every $24 \mathrm{hr}$ (RPL). Lactating female and the partition were removed on 1 a!l $2 \mathrm{C}$ at which time the litters were reconstituted to be horim. geneous by sex. From age 35 days on exercising hainster were housed in individual boxes with $23-25 \mathrm{~cm}$ dis

Eleven laboratory-reared hamsters were grven aliss ! individual dise exercisers at the age of 42 days for a period of 34 days. Ten hamsters of commarable starting weighi remained sedentary in suspended cages. There wer: bur females in each group. Two-hundred and seventy ardul: hamsters were assigned in equivalent numbers to excroisc in sedentary condition and were studied at differen! times over a 24 -month period. All but 4 of the adult hamsitrs were female and weighed between 80 and $180 \mathrm{~g}$ at the stam of the experiments.

Mean activity levels of the baboratory-reared inimal were determined as a function of their age between $n_{i 1} \times 14$ and 50 . In animals older than 5 weeks average daily activit? levels throughout the exposure to exercise were calculated for individual hamsters. The relationship between activit! levels and age or body weight was explored by calcuitting least-squares linear regressions for activity levels as function of age or of starting body weight, respectively

Ponderal growth rates of adult hamsters as a function of exercise or sedentary existence were obtained from the slopes of the least-squares linear regressions of weight increments over a $3-5$ week period of time. The relationship of growth rates and increases in body ucigh were then examined by plotting the growth rates of exercising and of sedentary hamsters as a function of the body weight registered on the first day of evercise. Ponderal growth velocities were alculated from weight changes in exercising and sedentary hamsters registered during 16 successive weekly intervals in 86 laboratory-horn. animals. Growth rates of exercising and sedentary hamstirs were also compared by plotting the growth rates of exercising hamsters as a function of growth rates displavod by their weight-matched sedentary controls.

Standard errors of the mean and of the estimale were used to express the variance in the averaged data and in th: linear regressions, respectively. Student's $t$ test and Pearson product coefficient of correlation wers used for statisticial evaluation of averages and linear regressions, respectively i regression analysis, general linear test $\{14\}$ was performed to compare the two functions relating the ponderal growth rates of exercising and sedentary hamsters as a function if increasing starting body weight.

\section{RESUITS}

Changes in the Activity Levels as a Function of Age

Young hamsters were observed to activate dises as soon as their eyes opened around the fifteenth day of life. By Day 19 some litters slept in the playground area in preference to the home compartment. Activity levels remained below 1,000 RPD until the age of 20 days. Between Days 20 and 35 the levels of voluntary disc activity increased by about 1,700 RPD (Fig. 1). The steep increase in the activity level of hamsters between the age of 20 and 38 days is defined by the linear regression $y=1846$ 


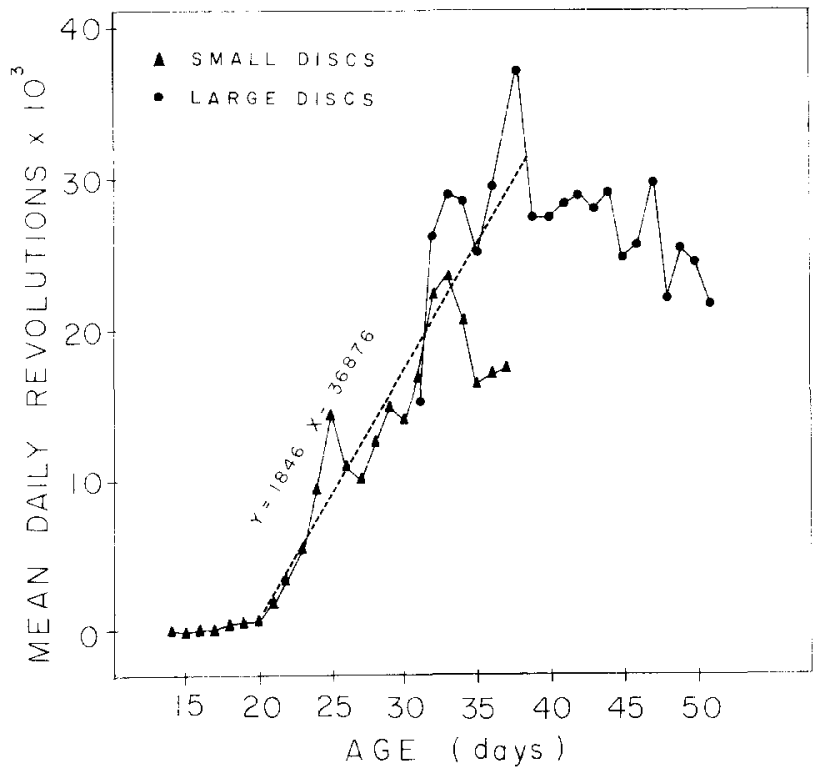

FIG. 1. Mean activity levels generated by 35 hamsters at ages 17 to 49 days on small-diameter dises in communal boxes (triangles) and on large-diameter discs in individual boxes (circles).

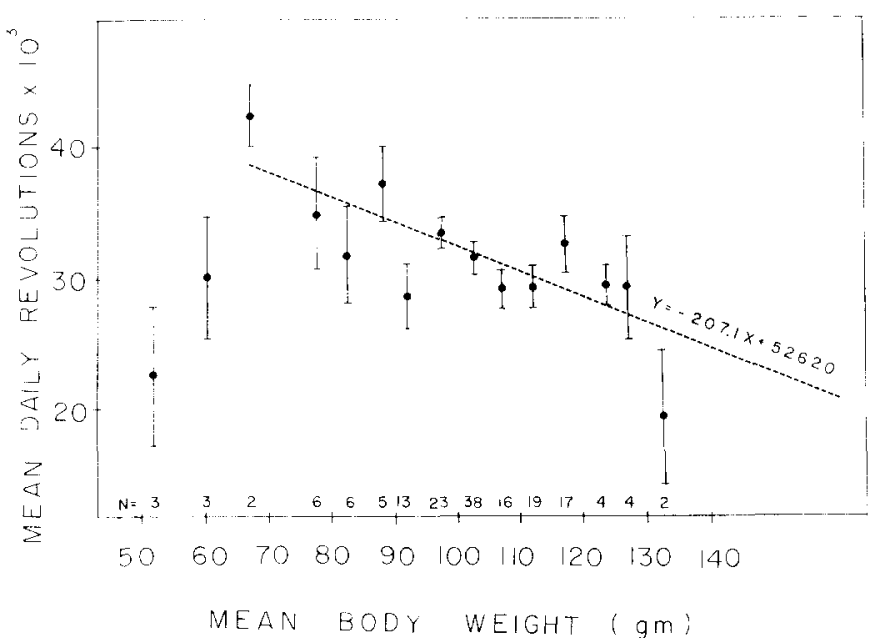

FIG. 2. Mean daily activity levels over a 34-day period of 137 adult and 24 laboratory-reared hamsters as a function of variable starting body weights.

$x-36876$ (SE EST $=2728.7, r=0.96)$. Around the fifth week of age the levels of voluntary activity plateau between 15,000 and 20,000 RPD in the communal boxes and between 25,000 and 35,000 RPD in the individual boxes. This difference could have resulted from the variable effectiveness of discs of different sizes, from the rate limiting effects of three dises with respect to a litter of six hamsters, or from antagonistic social behaviors observed in communal boxes from Day 19 on. There was no sex difference in the activity levels.

Changes in the Activity levels as a Function of Bod. Weight

The relationship between the mean activity levels of 137 adult and 24 laboratory-born hamsters and their starting body weights is presented in Fig. 2. At body weights between 50 and $70 \mathrm{~g}$ there is a rise in activity levels.

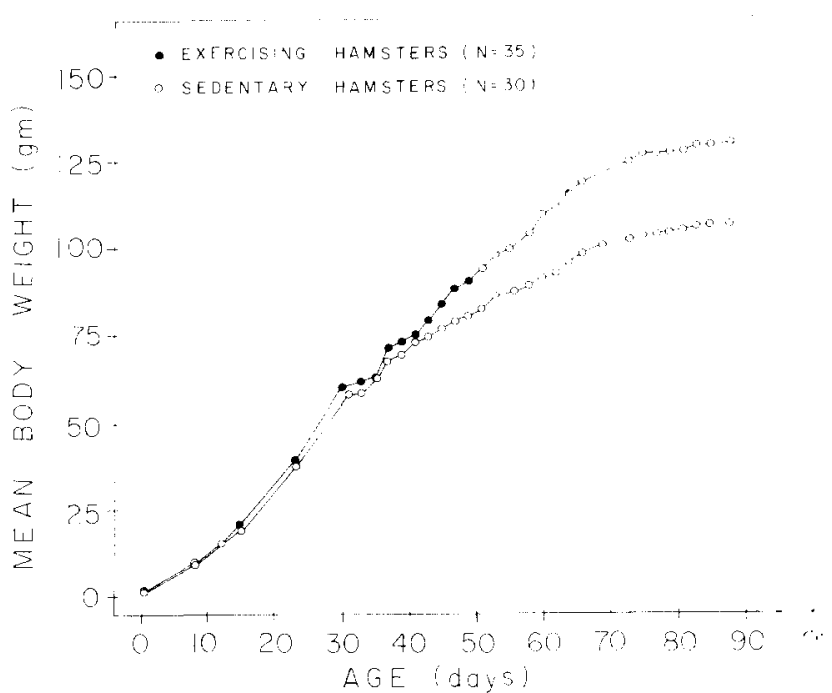

FIG. 3. Mean weight changes of 30 sedentary hamsters copen circles) and 35 hamsters given access to horizontal disc exercisers at the age 17 to 49 days (solid circles).

Activity levels decline at body weights above $70 \mathrm{~g}$ at the rate of about 2070 RPD for each $10 \mathrm{~g}$ increment in hody weight. This inverse relationship between hamster body weights above $65-70 \mathrm{~g}$ and the mean activity levels is described by the linear regression $y=207.1 x+52620$ (S) $\mathrm{EST}=3450.1, r=0.78$ ).

Delayed Acceleration of Growth b. Exercise During the Exponential Phase of Growth.

Weight changes of hamsters between birth and 90 (lits of life are presented in Fig. 3. During the first 5 weeks of life hamsters gain weight at rates of about $2 \mathrm{~g} / \mathrm{day}$. This exponential growth phase is modified by an inflexion point at age 30-40 days and body weight of $60-70 \mathrm{~g}$ at which time the growth rate changes to about $1 \mathrm{~g} / \mathrm{day}$. An additional decline in growth rate to about $0.3 \mathrm{~g} / \mathrm{day}$ takes place at age 70 days and body weight of about $100 \mathrm{~g}$. Exposure to exercisers at age 15 to 49 days had no influence over ponderal growth as long as hamsters grew at exponential rates. Between Days 35 and 70 . which included the last 14 days of exercise and the first 21 days of retirement, exercising hamsters gained $1.8 \mathrm{~g} /$ day while sedentary hamsters gained only about $1.0 \mathrm{~g} /$ day. Thus, the acceleration of ponderal growth by exercise became obvious at the age and body size at which hamsters first entered the asymptotic phase of growth. Following the 70 th day of life the retired exercised hamsters assumed the same asymptotic growth rate of about $0.3 \mathrm{~g} / \mathrm{day}$ displayed by their sedentary controls. On the 88 th day of life the retired exercised hamsters were significantly heavier $(1.30 .8$ \pm 3.2 vs $106.2 \pm 2.7 \mathrm{~g}, p<0.01)$ and significantly longer $(17.7 \pm 0.1$ vs $16.9+0.1 \mathrm{~cm}, p<0.01)$ than the sedentary hamsters but did not differ in the percentage of body fiat $(19.3 \pm 0.9$ vs $18.0 \pm 0.8 \%)$.

Growth Acceleration in Exercising Ilamsters During the Early Asymptotic Growth

Weight changes of the second group of laboratory-reared hamsters between ages 26 and 110 days are presented in Fig. 4. The inflexion from exponential to asymptotic growth rate of $0.2 \mathrm{~g} /$ day became apparent at the age of 


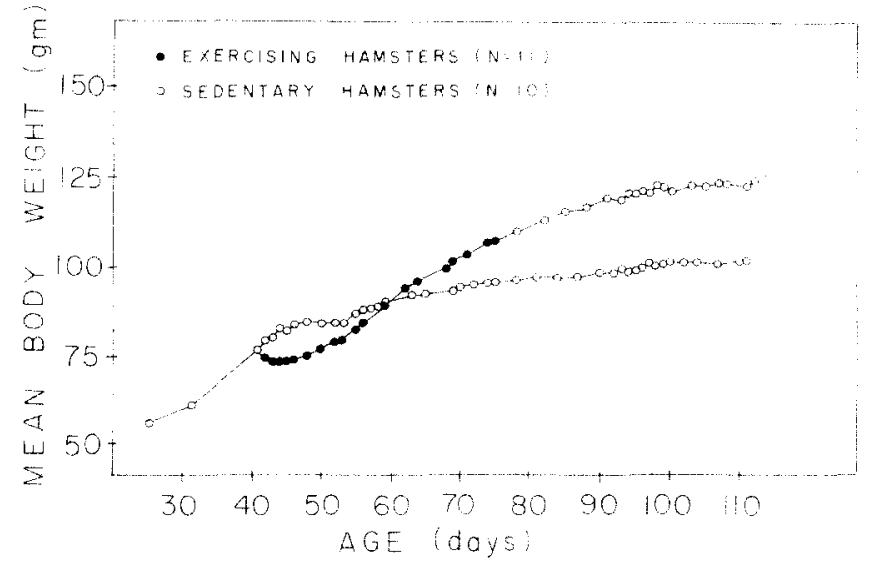

FIG. 4. Mean weight changes of 10 sedentary hamsters (open circles) and 11 hamsters with access to horizontal disc exercisers at the age 42 to 76 days (solid circles).

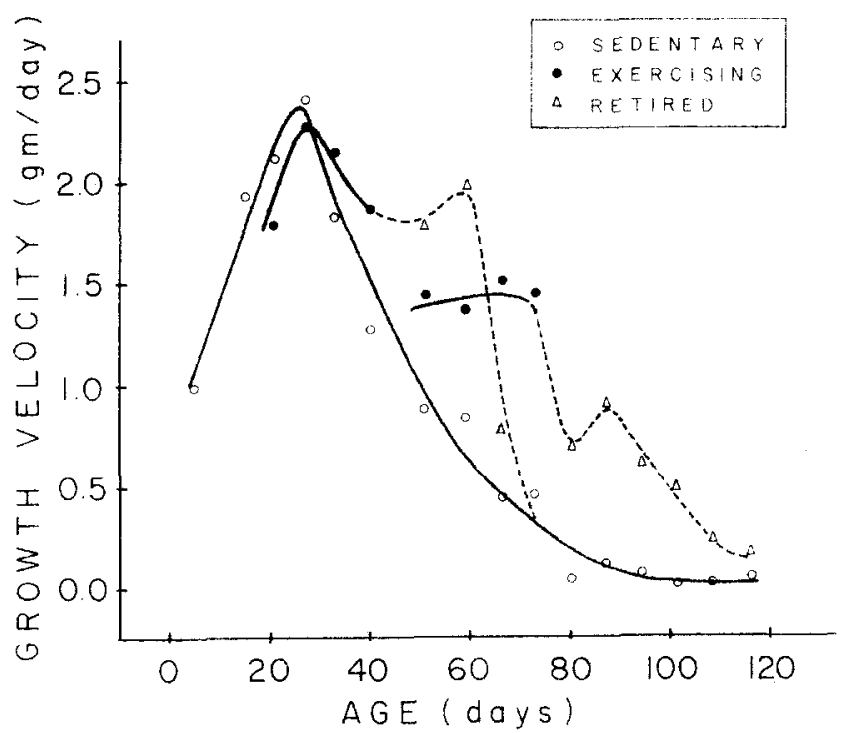

FIG. 5. Mean growth velocities of 86 laboratory-born hamsters as a function of age, of sedentary condition (open circles, $n=40$ ), or exercise (solid circles, $n=46$ ), or of the retirement from discs (triangles).

about 45 days and at body weights between 70 and $80 \mathrm{~g}$. During all but the first 4 days of exercise and during the first 10 days of retirement hamsters with access to exercisers on Days 42-76 gained about $1.2 \mathrm{~g} / \mathrm{day}$ in contrast to the low asymptotic growth rates of sedentary hamsters. Following the 85 th day of life the retired exercised hamsters assumed the low asymptotic growth rate exhibited by their sedentary controls. Unlike the previous group of hamsters which started exercise at an earlier age, the delay between the initiation of exercise on Day 42 and the onset of exercise-induced growth about Day 46 was very short. As in the younger group of exercising hamsters the onset of exercise-induced growth coincided with the onset of asymptotic growth in sedentary hamsters. On Day 110 of life the retired exercised hamsters were significantly heavier $(125.0$ \pm 3.4 vs $102.9 \pm 5.8 \mathrm{~g}, p<0.01)$ and longer $(17.7 \pm 0.2$ vs $16.7 \pm 0.9 \mathrm{~cm}, p<0.01)$ than the sedentary hamsters but did not contain a greater percentage of body fat (18.0 \pm 2.1 vs $16.1 \pm 2.7 \%$ ).

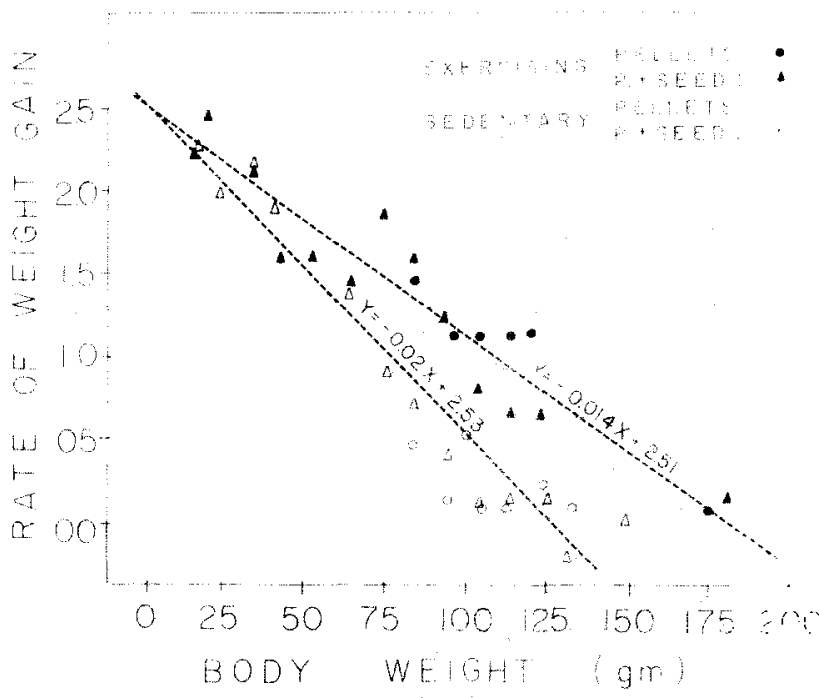

FIG. 6. Mean ponderal growth rates over a 3 to 5 -week period as : function of body weight at the time exercise was initiated. Diets consisted of pellets only (circles), or of pellets and sunflower seeds (triangles). One-hundred twenty-four adult and 46 laboratory-reared hamsters were exposed to exercise (solid symbols), while 75 adult and 32 laboratory-reared hamsters remained sedentary ropen symbols)

Changes in Ponderal Growth Velocities of Sedentary and Exercising Hamsters as a Function of Age

Changes in ponderal growth rates as a function of age are presented as ponderal growth velocities of sedentary and exercising hamsters in Fig. 5. In sedentary hamsters ponderal growth velocity rises to a maximum of about 2.4 $\mathrm{g} /$ day at the age of about 30 days, declines to about 0.2 $\mathrm{g} /$ day between Days 30 and 90 , and asymptotes between 0.2 and $0.1 \mathrm{~g} / \mathrm{kay}$ after about 90 days. Exercise is seen to increase the growth velocities above sedentary levels only after hamsters have entered the declining limb of the growth velocity curve. At ages above 30 days exercise appears to counteract the negative correlation between the growth velocity and age. Growth velocity remained steady and elevated throughout the period of exposure to discs in hamsters exercising at the age of $42 \cdots 76$ days.

Changes in Ponderal Growth Rates as a Function of Body. Weight

Figure 6 presents the growth rates of 124 adult and 40 laboratory-reared hamsters during their exposure to exercisers and of 75 adult and 32 laboratory-reared sedentary hamsters as a function of $5 \mathrm{~g}$ increments in their starting body weights. Two sets of growth rates were obtained from laboratory-reared hamsters, one for the 2 to 5 -week age block and the other for the 5 to 10 -week age block. Since sedentary animals with access to sunflower seeds wer. observed to sustain higher growth rates than hamsters with access to a diet of pellets alone, the data for the two dietary conditions are plotted separately in Fig. 6 . There is at inverse relationship between the rates of ponderal growth and the hamster body weight. The increasing body weight is associated with greater inhibition of ponderal growth rate in sedentary than in exercising hamsters. Linear regressions for growth rates as a function of body weight are $y=$ $0.020 x+2.53(\mathrm{SE}$ EST $=0.27, r=0.095)$ for 


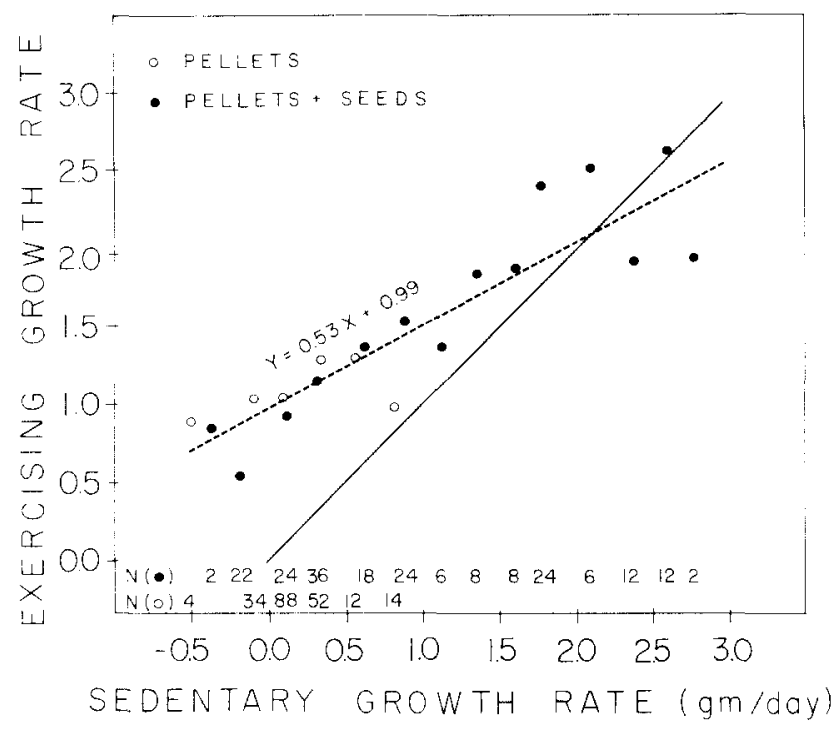

FIG. 7. The relationship between the growth rates of 204 exercising hamsters presented as a function of the growth rates exhibited by an equivalent number of weight-matched sedentary controls (broken

line). Solid line indicates points of equivalence on the two axes.

sedentary hamsters and $\mathrm{y}=-0.014 \mathrm{x}+2.51$ (SE EST $=$ $0.21, r=-0.94)$ for exercising hamsters. The regressions have a common intercept at about $2.5 \mathrm{~g} /$ day which is close to the highest observed exponential growth rates in this study. Both the regressions and their slopes are significantly different ( $\mathrm{F}$ values $33.06, p<0.0001$ and $11.14, p<0.002$, respectively for 1 and 34 degrees of freedom). Exercise attenuates the magnitude of the negative correlation of body weight over growth rate by reducing the slope of the regression line from -0.020 to -0.014 .

\section{Prediction of Exercise-Induced Growth Rates from Growth} Rates Observed in Sedentary Hamsters

The increase in hamster body weight is associated with a decline in ponderal growth. Disc exercise attenuates this relationship. If one were to relate the magnitude of exercise-induced growth to the rate of growth displayed by sedentary hamsters one could predict in weight-matched hamsters the extent to which exercise can be expected to accelerate growth. This type of relationship is presented in Fig. 7, where the growth rates of 124 adult and 40 laboratory-reared exercising hamsters are plotted as a function of growth rates displayed by the same number of weight-matched sedentary hamters. Double set of growth data were used for laboratory-reared hamsters, one for the 2 to 5 -week period and the other for the 5 to 10 -week period adding to a total of 204 exercising and 204 sedentary growth rates. The linear regression describing the growth rates in exercising hamsters as a function of growth rates in sedentary hamsters $y=0.53 x+0.99($ SE EST $=$ $0.27, r=0.90$ ) intersects the equivalence line at about 2.1 g/day. At sedentary growth rates of about $2.1 \mathrm{~g} /$ day exercise has no effect over ponderal growth in hamsters. As sedentary growth rates decline to zero, voluntary exercise exerts a progressively greater growth-accelerating effect. When ponderal growth rate reaches zero dise exercise induces the greatest relative acceleration in ponderal growth rate of about $0.99 \mathrm{~g} /$ day.

\section{DISCUSSION}

In the present study we have defined the portion of hamster ontogeny during which animals engage in voluntary activity on horizontal discs and the circumstances which are necessary for this type of exercise to accelerate ponderal and linear growth. Hamsters first start running on discs around Day 15, shortly after their eyes open. Daily activity levels undergo a rapid increase at the age 20 to 35 days 10 reach values of about 30,000 RPD. At body weights above $65 \mathrm{~g}$ activity levels decline by about 2,000 RPD for each $10 \mathrm{~g}$ increase in body weight. In both the age of onset of activity and the age of acceleration of activity levels hamsters are considerably more precocious than the rats. Rats first initiate voluntary activity in rotating drums at agc 40 to 70 days and accelerate their activity levels after Day 70 of life [18]. Voluntary disc activity in hamsters is characterized by an early onset, by very high daily rates of running, and by a progressive decline in activity levels as a function of increasing body weight.

We have found that the portion of hamster ontogeny associated with exercise-induced growth is much shorter than the portion of hamster life span supporting voluntary disc activity. Disc activity first leads to ponderal and lincir growth in excess of that displayed by sedentary hamsters at the age and body weight signalling the onset of asymptotic growth. An inflexion point from exponential growth rates in excess of $2 \mathrm{~g} /$ day to asymptotic growth rates of less than $1 \mathrm{~g} /$ day takes place at the age of $30-40$ days and hody weight of about $65 \mathrm{~g}$. An additional decline in growth rafe to about $0.3 \mathrm{~g} /$ day takes place at the age of about 70 days and body weight of about $100 \mathrm{~g}$. If exercise is initiated at an early time during the exponential phase of growth (fig. 3 ), the exercise-induced acceleration of growth is delayed until the time of onset of asymptotic growth in sedentary hamsters. If the onset of disc exercise and the onser of asymptotic growth coincide, the acceleration of ponderal and linear growth takes place without a long delay. In adult hamsters in whom exercise is initiated several wocks following the onset of asymptotic growth, disc excrcise leads to prompt acceleration of linear and ponderal growth [2]. It appears, therefore, that disc exercise does not superimpose an abnormal acceleration of growth over the preexisting endogenous growth rates. Instead, disc excrcise appears to reinstate some of the growth velocity which had undergone a spontaneous decline with increasing age and body size. In effect, disc exercise becomes relatively more effective in accelerating growth rates above sedentary level as the endogenous growth rates approximate zero velocity (Figs. 7 and 5).

What is the explanation for the negative relationship between hamster growth rates and increases in body sice (Fig. 6)? In part, this inverse relationship reflects it progressive loss of multiplicative capacity within the growing and differentiating organism. The slopes of lincar regressions of the growth rate over weight were negative for exercising as well as sedentary hamsters (Fig. 6). Thus, as hamsters grew larger they inevitably grew less rapidly, and disc exercise induced progressively lower absolute rates of growth. On the other hand, the fact that exercise was effective at all in inducing higher growth rates in hamsters points to the operation of some inhibitory influence over growth as hamsters grow larger in size. This negative relationship between the growth rates and some concomitant of increasing body size may be viewed as the 
outcome of some type of cumulative negative feedback. Whatever the nature of this inhibitory influence, it is apparently rendered less effective throughout the exposure to disc exercise and for a period of time following the termination of exercise (Figs. 5 and 6). Not only are the growth rates elevated throughout disc exercise, but the progressive decline of growth velocity with time appears to be counteracted (Fig. 5).

If the time of onset of endogenous growth suppression determines the time of onset of exercise-induced growth in early hamster ontogeny, why does disc exercise fail to accelerate growth at body weights above $180 \mathrm{~g}$ ? A plausible hypothesis is that exercise-induced growth takes place only when activity levels exceed a certain threshold level. At body weight of $180 \mathrm{~g}$, when disc exercise first fails to accelerate growth (Fig. 6) hamsters generate only about 15,000 RPD (Fig. 2). Early in ontogeny, the same levels of activity are attained at age of 28 days and precede by several days the first appearance of exercise-induced growth. Thus, the portion of ontogeny during which disc exercise accelerates growth is distinguished by voluntary activity levels in excess of 15,000 RPD. It is possible that the changes necessary for attenuation of inhibitory influence of body weight over growth are produced only when hamsters generate over 15,000 RPD.

How does the exercise-induced alteration in hamster body size relate to the current notions on the mainliami. of weight constancy in adult rodents? Disc exercise lials in permanent increases in hamster body weight. These $11 \mathrm{tg} h$ : increments represent true ponderal and linear growth alls: than the accumulation of excess holv fat $([2,3)$, and the present study). After they reestabliw the asymptoti: at: of growth the retired hamsters engage in the normal s.eforse of their elevated weight setpoint |3| The concill \& long-term regulation of body weight should be broitleneil to include the mechanism for mantenance of constan! body size through suppression of ponderal and ancas growth in addition to the more commonly dischuse! mechanism for the regulation of body fat reserves 17 . . I l: anatomical substrates for the growth-suppressing and t... the fat-regulating mechanisms appeat to interdigitats. vill. semicircular $[12,13,17]$ and parasagittal $[6]$ knifecul: anterolateral to medial basal hypothalamus lead i, siol. ultaneous interference with both ize-regulatory and ai regulatory functions. It would seem reasonable to pontlilits: that, in animals capable of continued growth, a gmowis. suppressive mechanism would be integrated with a :il regulating mechanism to insure the observed long-worn: regulation of body weight and size in adult rodents. Th. capacity of disc exercise to selectively increase hanisti: body size may provide a valuable tool for further sluhl c.: mechanisms of weight and size constancy in adult milence

\section{REFERENCES}

1. Babineau, L.-M. and E. Pagé. On body fat and body water in rats. Can. J. Biochem Physiol. 33: 970-979, 1955.

2. Borer, K. T. Absence of weight regulation in exercising hamsters. Physiol. Behav. 12: 589-597, 1974.

3. Borer, K. T. and A. A. Kooi. Regulatory defense of the exercise-induced weight elevation in hamsters. Behav. Biol. 13: $301-310,1975$.

4. Dawson, A. B. The persistence of the cartilage plates in the long bones of the white rats. Anat. Rec. 27: 202, 1925 (Abstract).

5. Donaldson, H. H. and S. B. Conrow. Quantitative studies on the growth of the skeleton of the albino rat. Am.J. Anat. 26: $237-314,1919$.

6. Gold, R. M. and G. Kapatos. Delayed hyperphagia and increased body lengths after hypothalamic knife cuts in weanling rats. J. comp. physiol. Psychol. 88: 202-209, 1975.

7. Kennedy, G. C. The role of depot fat in the hypothalamic control of food intake in the rat. Proc. Roy. Soc. Lond. B 140: $578-592,1953$.

8. Kennedy, G. C. The development with age of hypothalamic restraint upon the appetite of the rat. $J$. Endocr. 16: 9-17, 1957.

9. Kennedy, G. C. Interactions between feeding behavior and hormones during growth. Ann. N. Y. Acad. Sci. 157: 1049-1060, 1969.

10. Kennedy, G, C. and J. Mitra. Body weight and food intake as initiating factors for puberty in the rat. J. Physiol. 166: $408-418,1963$.

11. Kodama, A. M. In vivo and in vitro determinations of body fat and body water in the hamster. J. appl. Physiol. 31:218-222, 1971.
12. Mitchell, J. A., M. Hutchins, W. J. Schindler and V. Critchlow. Increase in plasma growth hormone concentration and nasoanal length in rats following isolation of medial bisal hypothalamus. Neuroendocr. 12: $161-173,1973$.

13. Mitchell, J. A., R. Smyrl, M. Hutchins, W. J. Schindler and V. Critchlow. Plasma growth hormone levels in rats with increased naso-anal length due to hypothalamie surgery. Neuroendocr. 10: $31-45,1972$.

14. Neter, J. and W. Wasserman. Applied Linear Statistical Hodels. Homewood, Illinois: R. D. Irwin, Inc., 1974.

15. Monteiro, L. S. and D. S. Falconer. Compensatory grow th and sexual maturity in mice. Anim. Prodtc. 8: 179-192, 1966.

16. Pace, N. and E. N. Rathbun. Studies on body composition. Ili. The body water and chemically combined nitrogen content in relation to fat content. J. biol. Chem 158: 685 691, 1945.

17. Palka, Y., R. Liebelt and V. Critchlow. Obesity and increased growth following partial or complete isolation of ventromedial hypothalamus. Physiol. Behav. 7: 187-194, 1971.

18. Wang, G. H. The relation between "spontaneous" activity and oestrous cycle in the white rat. Comp. Psuchol. Monogr. 2: 1. 27, 1923.

19. Widdowson, E. M. and R. A. Mccance. Some eftects of accelerating growth. I. General somatic development. Presc. Roy. Soc. Lond. B 152: 188-206, 1960.

20. Zucker, $I$. Body weight and age as factors determining esirngen responsiveness in the rat feeding system. Behar. Biol 7 : $527-542,1972$. 